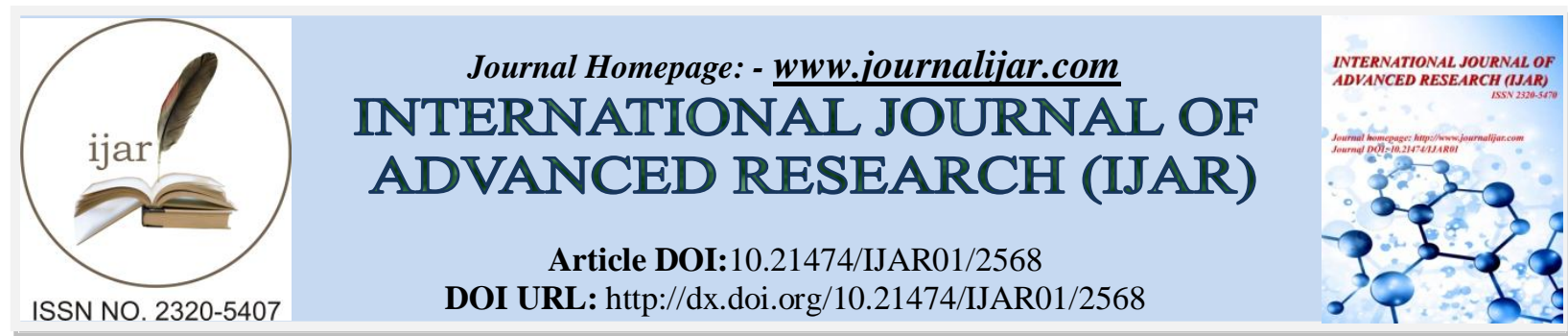

RESEARCH ARTICLE

\title{
FACTORS AFFECTING LONGEVITY OF TRICUSPID VALVE REPAIR.
}

Ahmed Al Turki ${ }^{1}$, Mohammad Marzogi ${ }^{2}$, Abdulaziz Naji ${ }^{3}$, Albader Al Hazmi ${ }^{4}$, Abdulaziz Alkhaldi ${ }^{5}$ and Abdelrahman Jamiel.

1. MD, Bachelor Degree of Respiratory Care from UOD, Dammam, KSA.

2. MD, Bachelor of Laboratory Medicine at UQU, Makkah, KSA.

3. MBBS, PT, Riyadh, Saudi Arabia.

4. MD, Bachelor of Clinical Laboratory Medicine from KSU, Riyadh, KSA.

5. Consultant cardio-thoracic surgery, king Abdulaziz medical city, Riyadh, KSA.

6. Consultant cardiology, king Abdulaziz medical city, Riyadh, KSA.

\section{Manuscript Info}

Manuscript History

Received: 27 October 2016

Final Accepted: 25 November 2016

Published: December 2016

\section{Abstract}

Background:Severe tricuspid regurgitation (TR) is an independent predictor of long-term mortality. Although TR is tolerated for many years, it will eventually lead to severe decompensated heart failure and death. The 1-year survival rate in patients with severe TR is $65 \%$, whereas that in patients without TR is $90 \%$.

Methods: This survival retrospective and prospective study included 199 patients (66 male [33.2\%] and 133 female [66.8\%]) who underwent tricuspid valve repair at King Abdulaziz Cardiac Center, Riyadh, Saudi Arabia, from January 1999 until December 2012. The mean age at operation was 49.7 years SD 16.3. All patients received follow-up until February 2016. We collected data on patient demographics, family history of cardiac diseases, smoking history, patient health status and other co-morbidities prior to the operation, the surgical technique, and other associated procedures carried out during the same operation. We included echocardiograph (ECHO)results obtained pre-operation, post-operation, pre-discharge, and at the final follow-up (February 2016). Cox regression analysis (univariate and multivariate) was used to study patient survival factors.

Results:The final outcomes of the operation showed $84.5 \%$ survival. The median survival time was 50 months and interquartile range 72 . All patients underwent concomitant procedures such as coronary artery bypass grafting $(19.1 \%)$ or other valve replacement surgeries (23.1\%). The surgical technique was chosen based on the patient's status and the surgeon's preference. The mean hospital stay was 26.9 \pm 19.3 days, which was prolonged by surgical complications. Elderly patients, prior cardiac surgery, and abnormal coronary angiogram were linked to poor prognosis $(\mathrm{p} \leq 0.05)$. ECHO follow-up showed improvement in the functional status of the tricuspid valve.

Corresponding Author:-Ahmed Al Turki.

Address:- Medical Intern, BachelorDegree ofRespiratory Care from UOD, Dammam, KSA. 
Conclusion: The tricuspid valve requires surgical intervention whenever possible so as to minimize fatal consequences. Our study revealed positive enhancement of valve function following surgery.

Copy Right, IJAR, 2016,. All rights reserved.

\section{Background:-}

Tricuspid regurgitation (TR) is increasingly recognized as a significant valvular disorder with serious clinical consequences. Severe TR is an independent predictor of long-term mortality. Although TR is tolerated for many years, it will eventually lead to severe decompensated heart failure and death. The 1-year survival rate in patients with severe TR is $65 \%$, whereas that in patients with no TR is $90 \%$.[1] In clinical practice, there is marked inconsistency regarding indications, timing, and techniques of tricuspid valve repair, with variability in the final outcome. Gatti et al. reported an improvement in tricuspid valve dilatation after surgery, with respect to TR, with the estimated freedom from all-cause death being $77.8 \%$ for 10 years. [2] McCarthy et al. reported the experience of the Cleveland Clinics in tricuspid valve repairs in 2004. They showed a high level of early recurrence of significant TR (14\%) a week after tricuspid valve repair. In addition, their results suggested that the risk factors for worsening regurgitation are higher pre-operative regurgitation grade, poor left ventricular function, permanent pacemaker, and repair type other than ring annuloplasty.[3] In 2008, Guenther et al. reported their 30 years' experience with tricuspid valve surgery (repair and replacement). In their study, they reported a high (18.8\%) postoperative mortality for tricuspid valve surgery.[4] In 2010, Fadel determined the short- and long-term outcomes after triple valve replacement (aortic, mitral, and tricuspid valves). Their results for 206 patients showed that aortic and mitral valves usually underwent replacement $(>70 \%)$ and tricuspid valve usually underwent repair (91\%). The study showed that operation mortality prior to hospital discharge was $11 \%$ and the 15 -year survival rate was $68 \%$. In addition, they reported that advanced age was one of the major risk factors for high mortality.[5] In 2013, Al-Atassi et al. published a literature review that concluded according to the available evidence that severe tricuspid valve regurgitation should be repaired whenever possible.[6] Therefore, for patients with TR, it is important to consider at the time of decisionmaking the risk factors (modifiable and non-modifiable) that might influence the outcome of any planned surgical intervention.Moreover, surgeons need to individualize patient plans in order to improve the short- and long-term outcomes and to minimize the likelihood of recurrence of TR.

\section{Methodology:-}

This was a survival retrospective and prospective study including all cases of tricuspid valve repair done at King Abdulaziz Cardiac Center, Riyadh, Saudi Arabia from January 1999 until December 2012. Subjects included in this study were followed-up until February 2016. We collected data on patient demographics, family history of cardiac diseases, smoking history, patient health status together with other co-morbidities prior to the operation, echocardiographic (ECHO) findings (pre- and post-operation), surgical techniques, and other associated procedures performed during the same operation. This information was obtained from the Cardiac Surgery Database, in addition to direct patient chart review. Patients were followed-up postoperatively with ECHO during regular hospital visits. Visits were scheduled according to patient need and physician preference. We included and compared the ECHO carried out pre-operation, post-operation, pre-discharge, and at final follow-up. Cox regression analysis (univariate and multivariate) was used to study patients' survival factors. Final outcomes of changes in tricuspid valve across time and patient survival were reported. This study was approved by the institutional review board of King Abdullah International Medical Research Center, Riyadh, Saudi Arabia.

\section{Results:-}

We included 199 patients (66 male [33.2\%] and 133 female [66.8\%]) in this study. The mean age at operation was 49.7 years SD 16.3. The final outcomes of the operation showed $84.5 \%$ survival. The median survival time was 50 months and interquartile range 72 (Figure 1). Patients' characteristics are presented in Table I, and all co-morbidities are described in Table II. All patients underwent concomitant procedures such as coronary artery bypass grafting $(\mathrm{CABG})$, mitral valve repair/replacement, and/or aortic valve repair/replacement (Table III). The surgical technique selectedwas based on the patient's status and the surgeon's preference (Table IV). Some patients underwent a combination of techniques, but the effect of this was not measured. The mean hospital stay was $26.9 \pm 19.3$ days, which was prolonged in patients who experienced surgical complications (Table V). Patients who experienced arrhythmia, pneumonia, and/or other infections tended to stay in hospital for a longer period. Paired difference 
analysis tests were used to report the positive significant difference with regard to improvement of tricuspid valve function over time. The performance of the target tricuspid valve was followed up by ECHO (Table VI). Cox regression analysis (univariate \& multivariate) represents the survival rate (Table VII, Figure 2). Survival outcomes are summarized in Table VIII.

Table I:- Patient Characteristics

\begin{tabular}{|c|c|c|}
\hline Characters & $\mathrm{n}=199$ & $(\%)$ \\
\hline Male & 66 & 33.2 \\
\hline Female & 133 & 66.8 \\
\hline Family History of Cardiac Disease & 9 & 4.5 \\
\hline Smoker & 14 & 7 \\
\hline Mean Body Mass Index & & $27.08 \pm 7.45$ \\
Under-weight & 20 & 10.1 \\
Normal & 60 & 30.2 \\
Over-weight & 61 & 30.7 \\
Obese & 58 & 29.1 \\
\hline Abnormal Angiogram Finding & 149 & 74.9 \\
\hline Abnormal ECG Finding & 160 & 80.5 \\
\hline
\end{tabular}

Table ii:- co-morbidities.

\begin{tabular}{|c|c|c|}
\hline Disease & $\mathrm{n}=199$ & $(\%)$ \\
\hline Hypertension & 83 & 41.7 \\
\hline Diabetes Mellitus & 76 & 38.2 \\
\hline Ischemic Heart Disease & 35 & 17.6 \\
\hline Rheumatic Heart Disease & 88 & 44.2 \\
\hline Congestive Heart Failure & 44 & 22.1 \\
\hline Pulmonary Diseases & 31 & 15.6 \\
\hline Dyslipidemia & 59 & 29.6 \\
\hline Pulmonary Hypertension & 42 & 21.1 \\
\hline Chronic Kidney Disease & 15 & 7.5 \\
\hline Stroke & 19 & 9.5 \\
\hline
\end{tabular}

Table III: Associated Procedure

\begin{tabular}{|c|c|c|}
\hline Procedure \& Technique & $\mathrm{n}=199$ & $(\%)$ \\
\hline Mitral Valve Repair & 46 & 23.1 \\
\hline Mitral Valve Replacement & 125 & 62.8 \\
\hline Aortic Valve Replacement & 39 & 19.6 \\
\hline CABG & 38 & 19.1 \\
\hline Maze Procedure & 60 & 31.3 \\
\hline
\end{tabular}

Table IV: Surgical Technique

\begin{tabular}{|c|c|c|}
\hline Procedure \& Technique & $\mathrm{n}=199$ & $(\%)$ \\
\hline Kay repair & 114 & 57.3 \\
\hline De Vega & 22 & 11.1 \\
\hline Commissurotomy & 21 & 10.9 \\
\hline Ring & 46 & 23.1 \\
\hline
\end{tabular}

Table V: Hospital Stay

\begin{tabular}{|c|c|c|c|}
\hline Variable & Mean $( \pm$ SD) & $\mathrm{n}=199(100 \%)$ & P value \\
\hline Age & $49.71(16.35)$ & & 0.001 \\
\hline Gender & $27.02(23.57)$ & 66 & 0.97 \\
Male & $26.91(16.91)$ & 133 & \\
Female & $31.07(23.83)$ & 14 & 0.409 \\
\hline Smoking History &
\end{tabular}




\begin{tabular}{|c|c|c|c|}
\hline No & $26.62(19.02)$ & 185 & \\
\hline $\begin{array}{c}\text { Previous Cardiac Surgery } \\
\text { Yes } \\
\text { No }\end{array}$ & $\begin{array}{l}26.55(12.99) \\
27.15(21.99)\end{array}$ & $\begin{array}{c}66 \\
133\end{array}$ & 0.838 \\
\hline $\begin{array}{c}\text { Heart Failure } \\
\text { Yes } \\
\text { No }\end{array}$ & $\begin{array}{l}36.02(27.04) \\
24.36(15.74)\end{array}$ & $\begin{array}{c}43 \\
156\end{array}$ & 0.009 \\
\hline $\begin{array}{c}\text { Ischemic Heart Disease } \\
\text { Yes } \\
\text { No }\end{array}$ & $\begin{array}{l}31.29(21.30) \\
25.63(18.62)\end{array}$ & $\begin{array}{c}45 \\
154\end{array}$ & 0.08 \\
\hline $\begin{array}{c}\text { CABG } \\
\text { Yes } \\
\text { No }\end{array}$ & $\begin{array}{l}29.37(20.66) \\
26.35(19.06)\end{array}$ & $\begin{array}{c}38 \\
161\end{array}$ & 0.391 \\
\hline $\begin{array}{c}\text { Complicated by Pneumonia } \\
\text { Yes } \\
\text { No }\end{array}$ & $\begin{array}{l}47.72(40.42) \\
24.69(14.29)\end{array}$ & $\begin{array}{c}18 \\
181\end{array}$ & 0.028 \\
\hline $\begin{array}{c}\text { Complicated by Bleeding } \\
\text { Yes } \\
\text { No } \\
\end{array}$ & $\begin{array}{l}30.73(21.19) \\
26.33(19.12)\end{array}$ & $\begin{array}{c}22 \\
177\end{array}$ & 0.318 \\
\hline $\begin{array}{c}\text { Complicated by Infection } \\
\text { Yes } \\
\text { No } \\
\end{array}$ & $\begin{array}{l}35.69(20.46) \\
23.82(18.07)\end{array}$ & $\begin{array}{c}49 \\
150\end{array}$ & 0.000 \\
\hline $\begin{array}{c}\text { Complicated by Arrhythmia } \\
\text { Yes } \\
\text { No }\end{array}$ & $\begin{array}{l}29.16(18.11) \\
23.41(20.71)\end{array}$ & $\begin{array}{c}115 \\
84\end{array}$ & 0.043 \\
\hline \multicolumn{4}{|c|}{ Significant $\mathrm{P}$ value, $<0.05$} \\
\hline
\end{tabular}

Table VI: ECHO follow-up of the tricuspid valve

\begin{tabular}{|c|c|c|c|c|c|c|}
\hline Regurgitation grade & Trace & Mild & Moderate & Severe & Compere Test & $\mathrm{P}$ value \\
\hline Pre-Surgery & $3 \%$ & $4.2 \%$ & $47 \%$ & $45.8 \%$ & \multirow[t]{2}{*}{ Friedman's } & \multirow[t]{2}{*}{0.000} \\
\hline Post-Surgery & $43.2 \%$ & $25.9 \%$ & $19.4 \%$ & $11.5 \%$ & & \\
\hline \multirow[t]{4}{*}{$\begin{array}{l}\text { Valve Annular } \\
\text { Diameter }\end{array}$} & \multicolumn{2}{|c|}{$\begin{array}{l}\text { Mean Anterior-posterior } \\
\text { (Pre-Surgery) }\end{array}$} & \multicolumn{2}{|c|}{$\begin{array}{l}\text { Mean Anterior-posterior } \\
\text { (Post-Surgery) }\end{array}$} & & \\
\hline & \multirow{2}{*}{\multicolumn{2}{|c|}{$\begin{array}{c}3.71 \pm 0.72 \\
\begin{array}{c}\text { Abnormal T.A.P.S.E } \\
\text { (Pre-Surgery) }\end{array}\end{array}$}} & \multicolumn{2}{|c|}{$3.14 \pm 0.67$} & Shapiro-Wilk & 0.000 \\
\hline & & & $\begin{array}{r}\text { Abnorm } \\
\text { (Post }\end{array}$ & $\begin{array}{l}\text { A.P.S.E } \\
\text { gery) }\end{array}$ & & \\
\hline & \multicolumn{2}{|c|}{$32.7 \%$} & \multicolumn{2}{|c|}{$38.6 \%$} & Cochrane's Q & 0.142 \\
\hline \multicolumn{7}{|l|}{$\begin{array}{l}\text { Rt. Ventricular } \\
\text { Dilatation }\end{array}$} \\
\hline & Fraction Area Change & Mild & Moderate & Severe & & \\
\hline Pre-Surgery & & $77 \%$ & $17.7 \%$ & $4.9 \%$ & \multirow[t]{2}{*}{ Friedman's } & \multirow[t]{2}{*}{0.000} \\
\hline \multirow[t]{2}{*}{ Post-Surgery } & & $85.8 \%$ & $12.1 \%$ & $2.1 \%$ & & \\
\hline & RV Systolic Function & Normal & Mild & Moderate & & \\
\hline Pre-Surgery & & $70.9 \%$ & $12.7 \%$ & $16.3 \%$ & \multirow[t]{2}{*}{ Friedman's } & \multirow[t]{2}{*}{0.062} \\
\hline Post-Surgery & & $62 \%$ & $21.1 \%$ & $16.9 \%$ & & \\
\hline
\end{tabular}


Table VII: Cox Regression Survival Analysis

\begin{tabular}{|c|c|c|c|c|}
\hline Variable & Univariate Model & $P$ value & Multivariate Model & $\mathrm{P}$ value \\
\hline Age & $1.04(1.01,1.08)$ & 0.010 & $1.16(1.05,1.27)$ & 0.002 \\
\hline Gender & $0.83(0.29,2.31)$ & 0.722 & $0.34(0.03,3.26)$ & 0.355 \\
\hline Smoking History & $1.31(0.17,9.88)$ & 0.789 & $0.01(0.000,1.41)$ & 0.071 \\
\hline Weight & $0.96(0.97,1.02)$ & 0.740 & $0.98(0.94,1.03)$ & 0.565 \\
\hline Previous Cardiac Surgery & $5.20(1.19,22.70)$ & 0.028 & $49.1(3.55,678.8)$ & 0.004 \\
\hline Normal ECG & $1.17(0.47,2.91)$ & 0.722 & $6.25(0.71,54.7)$ & 0.098 \\
\hline Normal Angiogram & $2.69(0.62,11.66)$ & 0.185 & $45.2(3.01,678.7)$ & 0.006 \\
\hline Hypertension & $0.55(0.22,1.37)$ & 0.202 & $0.89(0.13,5.89)$ & 0.909 \\
\hline Heart failure & $0.43(0.17,1.11)$ & 0.084 & $0.63(0.10,3.89)$ & 0.624 \\
\hline Rheumatic Heart Disease & $1.41(0.55,3.60)$ & 0.464 & $7.79(1.05,57.7)$ & 0.044 \\
\hline Ischemic Heart Disease & $0.55(0.20,1.48)$ & 0.239 & $0.53(0.01,16.02)$ & 0.715 \\
\hline Pulmonary Hypertension & $0.61(0.22,1.73)$ & 0.363 & $0.09(0.01,0.96)$ & 0.047 \\
\hline Pulmonary Disease & $0.78(0.22,2.70)$ & 0.698 & $1.11(0.17,6.98)$ & 0.911 \\
\hline Dyslipidemia & $0.57(0.22,1.46)$ & 0.245 & $0.77(0.11,5.37)$ & 0.798 \\
\hline Diabetes Mellitus & $1.02(0.39,2.65)$ & 0.959 & $42.7(2.54,719.2)$ & 0.009 \\
\hline Stroke & $0.93(0.21,4.09)$ & 0.931 & $0.10(0.003,3.22)$ & 0.196 \\
\hline Chronic Kidney Disease & $0.24(0.07,0.74)$ & 0.014 & $0.01(0.000,0.66)$ & 0.029 \\
\hline Maze Procedure & $0.79(0.30,2.05)$ & 0.635 & $0.14(0.02,0.84)$ & 0.032 \\
\hline Kay Repair & $1.36(0.54,3.44)$ & 0.507 & $3.67(0.75,17.8)$ & 0.106 \\
\hline De Vega & $0.43(0.14,1.33)$ & 0.147 & $0.49(0.07,3.28)$ & 0.469 \\
\hline Commissurotomy & $1.007(0.23,4.38)$ & 0.993 & $0.98(0.08,11.3)$ & 0.993 \\
\hline Mitral Repair & $0.64(0.22,1.81)$ & 0.404 & $13.3(0.57,311.7)$ & 0.106 \\
\hline Mitral Replacement & $1.28(0.49,3.32)$ & 0.610 & $9.27(0.43,196.1)$ & 0.153 \\
\hline Aortic Replacement & $0.63(0.22,1.76)$ & 0.379 & $0.03(0.004,0.39)$ & 0.006 \\
\hline CABG & $0.57(0.20,1.62)$ & 0.298 & $1.24(0.05,29.7)$ & 0.891 \\
\hline Complicated by Pneumonia & $0.43(0.12,1.50)$ & 0.188 & $0.07(0.005,1.17)$ & 0.065 \\
\hline Complicated by Bleeding & $0.89(0.20,3.87)$ & 0.877 & $0.97(0.05,16.8)$ & 0.984 \\
\hline Complicated by Infection & $0.48(0.18,1.25)$ & 0.135 & $0.34(0.05,2.07)$ & 0.244 \\
\hline Complicated by Arrhythmia & $1.005(0.38,2.59)$ & 0.992 & $0.55(0.08,3.59)$ & 0.534 \\
\hline
\end{tabular}

Table viii: survival time

\begin{tabular}{|c|c|c|}
\hline Period & $\mathrm{N}=199$ & $(\%)$ \\
\hline One Month & 177 & 88.9 \\
\hline One Year & 146 & 73.4 \\
\hline Five Years & 78 & 39.2 \\
\hline Ten Years & 24 & 12.1 \\
\hline
\end{tabular}




\section{Survival Time per month}

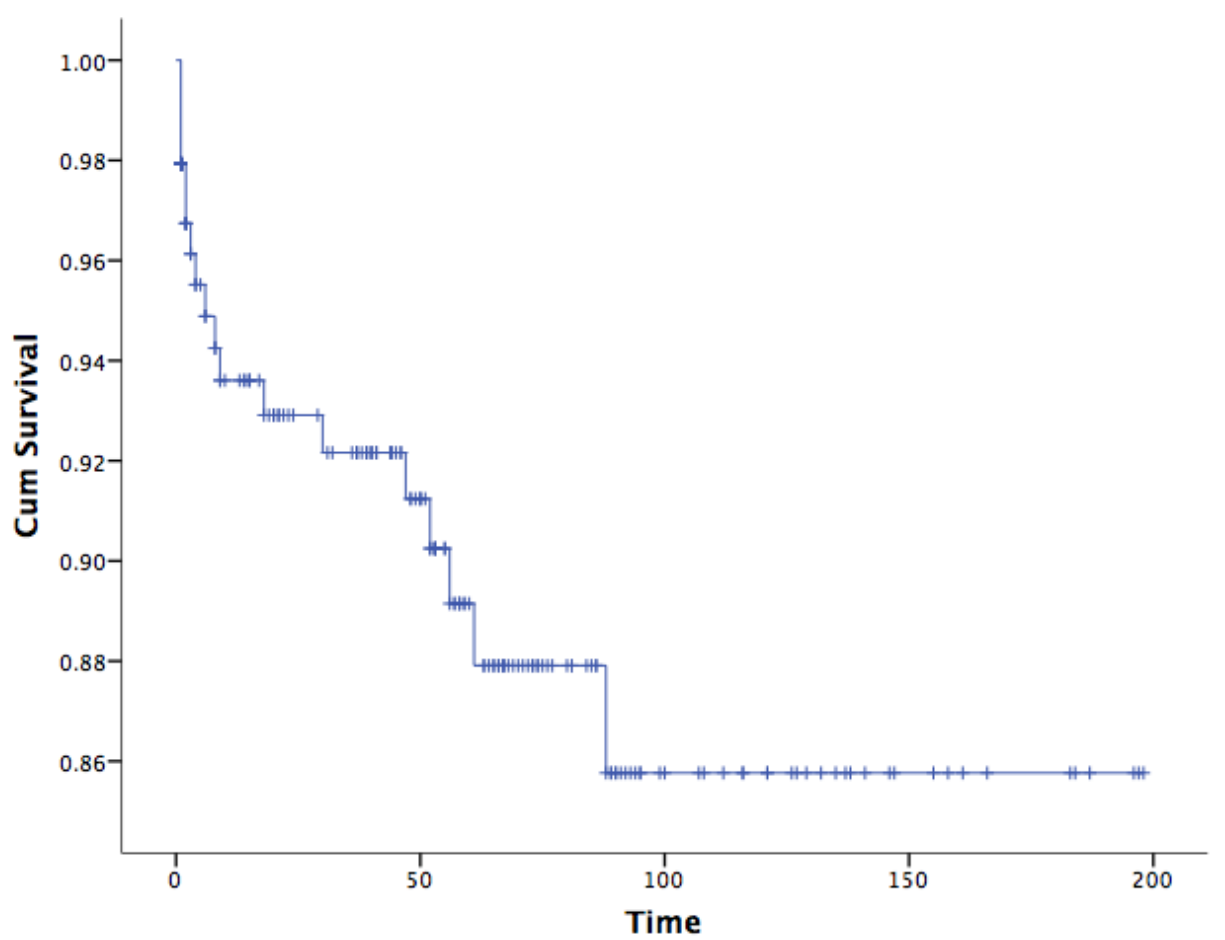

Figure 1:-

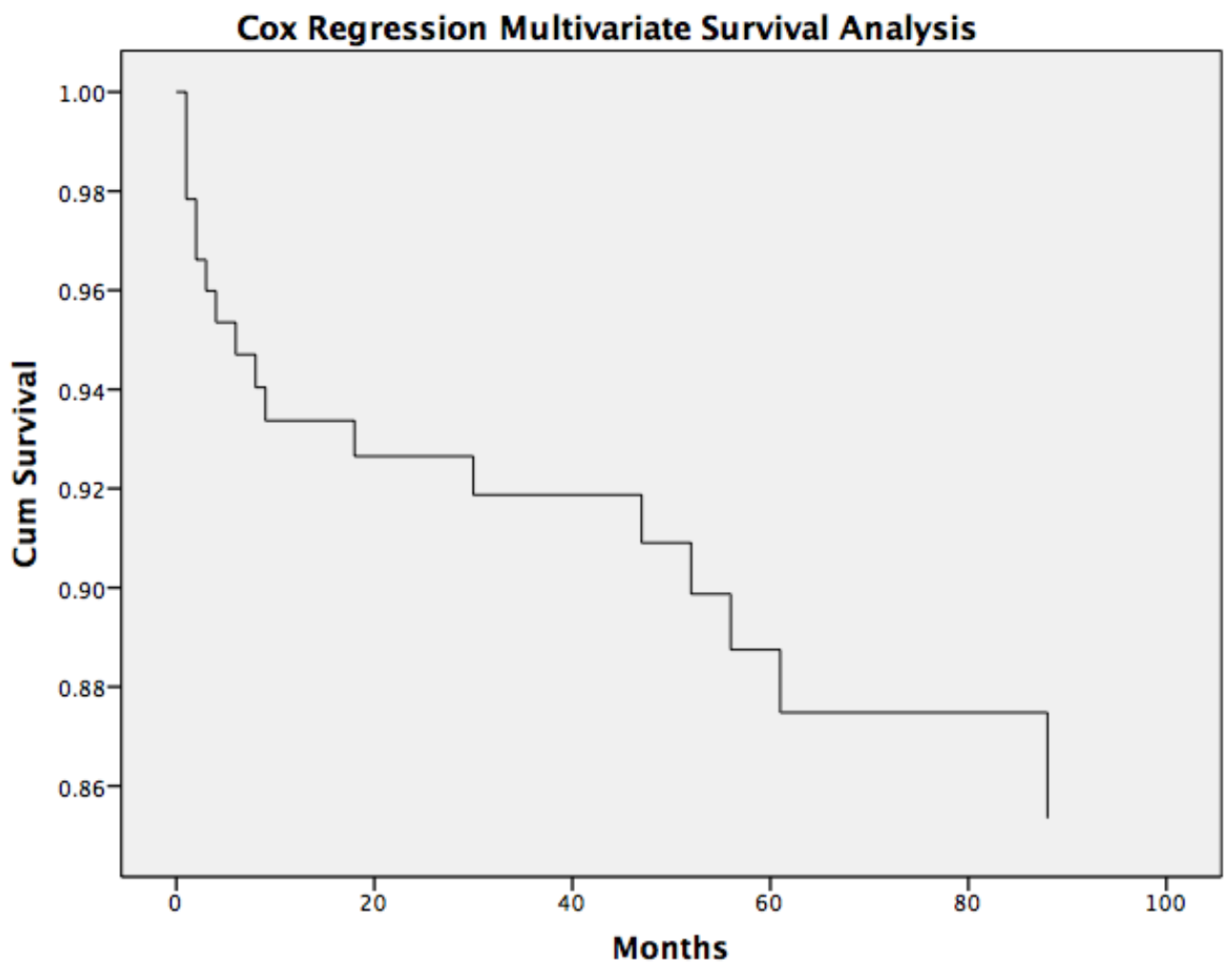

Figure 2:- 


\section{Discussion:-}

Our study showed an excellent outcome for patients who underwent tricuspid valve surgery. Our results were consistent with a 2013 review by Al-Atassi et al., who strongly recommended tricuspid valve repair whenever possible.Their recommendation was based on a definite improvement in patient status. ${ }_{[6]}$. To the best of our knowledge, this is the first study from Saudi Arabia that addresses this topic; therefore, we do not have any local data for comparison. In clinical practice, clinical improvement in patient status is the target achievement of tricuspid valve surgery. This is primarily monitored by ECHO data, according to a review paper published by Huttin et al., in 2016.[7] We followed the same regimenwith our patients, who were monitored after surgery by frequent ECHO studies that were interpreted by a certified cardiologist. This should lead to a definite enhancement in patient care, and should diagnose any recurrence at an early stage. A meta-analysis conducted by Kara et al. in 2015 included 2,488 patients from 10 studies and revealed a significant reduction of tricuspid valve disease progression in those who underwent a surgical repair.[8]

Unfortunately, we did not consider the cause of death, which would have provided more information about risk factors for survival. Patients who were re-operated on survived. Time and cause for re-operation were not measured.

\section{Conflicts of Interest:-}

The author(s) of this publication has no research support from any source. This project was reviewed and approved by the King Abdullah International Research Center, Riyadh, Saudi Arabia, in accordance with its research policy.

\section{References:-}

1. Nath J, Foster E, Heidenreich PA. Impact of tricuspid regurgitation on long-term survival. J Am Coll Cardiol. 2004 Feb 4;43(3):405-9.http://www.ncbi.nlm.nih.gov/pubmed/15013122

2. Gatti G, Dell'angela L, Morosin M, et al. Tricuspid annuloplasty for tricuspid regurgitation secondary to leftsided heart valve disease: immediate outcomes and risk factors for late failure. Can $\mathrm{J}$ Cardiol. 2015; http://www.ncbi.nlm.nih.gov/pubmed/26777269

3. McCarthy PM, Bhudia SK, Rajeswaran J, Hoercher KJ, Lytle BW, Cosgrove DM, et al. Tricuspid valve repair: durability and risk factors fo failure. J Thorac Cardiovasc Surg. 2004 Mar;127(3):67485.http://www.ncbi.nlm.nih.gov/pubmed/15001895

4. Guenther T, Noebauer C, Mazzitelli D, Busch R, Tassani-Prell P, Lange R. Tricuspid valve surgery: a thirtyyear assessment of early and late outcome. Eur J Cardio-Thorac Surg Off J Eur Assoc Cardio-Thorac Surg. 2008 Aug;34(2):402-9; discussion 409.http://www.ncbi.nlm.nih.gov/pubmed/18579403

5. Fadel BM, Alsoufi B, Manlhiot C, McCrindle BW, Siblini G, Al-Halees Z, et al. Determinants of short and long-term outcomes following triple valve surgery. J Heart Valve Dis. 2010 Jul;19(4):513-523; discussion 523.http://www.ncbi.nlm.nih.gov/pubmed/20845901

6. Al-Atassi T, Toeg H, Chan V, Ruel M. Current readings: status of tricuspid valve repair. Semin Thorac Cardiovasc Surg. 2013;25(1):30-7.http://www.ncbi.nlm.nih.gov/pubmed/23800527

7. Huttin O, Voilliot D, Mandry D, Venner C, Juillière Y, Selton-suty C. All you need to know about the tricuspid valve: tricuspid valve imaging and tricuspid regurgitation analysis. Arch Cardiovasc Dis. 2016;109(1):6780.http://www.ncbi.nlm.nih.gov/pubmed/26711544

8. Kara I, Koksal C, Erkin A, et al. Outcomes of Mild to Moderate Functional Tricuspid Regurgitation in Patients Undergoing Mitral Valve Operations: A Meta-Analysis of 2,488 Patients. Ann Thorac Surg. 2015;100(6):2398407.http://www.ncbi.nlm.nih.gov/pubmed/26525867 